more definitive information on translation initiation in yeast.

Further speculation must await additional key findings. However it is already clear that the DNA region upstream from

Charles Yanofsky is at the Department of Biological Sciences, Stanford University, Stanford, California 94305.
LEU2 has structural features resembling those of the regulatory regions of prokaryotic biosynthetic operons regulated by attenuation. Thus further studies with $L E U 2$ of yeast may reveal how a regulatory region evolved from a prokaryote has become modified to allow regulation appropriate to a eukaryote.

\title{
Stratigraphy
}

\section{Is the present long enough to measure the past?}

\section{from Peter M. Sadler}

As students of geological history, stratigraphers must deal routinely with time spans that are far longer than their experience. From evidence preserved in layered sedimentary rocks, radical longterm changes in the configuration of oceans and continents, ice-caps and mountains can be charted. But the present, in which we can observe such processes in action, is incomparably brief. Geological processes are typically erratic, and their short-term characteristics can be very poor guides to their long-term behaviour. Stratigraphy can therefore sometimes lead to results that seem counter-intuitive in the light of present-day observations of geological processes in action.

Consider the magnitude of the time-scale discrepancy between the past and present in stratigraphy. The cumulative record of Earth history, as contained in stratified sedimentary rocks, spans three billion years. The shorter local sequences of rocks that constitute that record commonly span millions and tens of millions of years. Furthermore, it is still rare for time correlations between sequences to be more precise than one million years. In the oldest parts of the record, precision is far worse; within the deposits of the last one million years it can be far better; and of course, the present and historic past are studied with much greater precision still.

What is the time span of the present? Few individuals have studied one deposition system for even a decade. Continuous monitoring of sedimentation is rare, and seldom maintained beyond a few hours. Indeed, actualism in geology - the study of the present as a means to understand the record of past processes - has been common for little more than a century, following the leads of Hutton, Lyell and Darwin.

Yet sedimentology and stratigraphy deal with quite incongruous time scales. The incompatibility comes sharply into focus when modern rates of sediment deposition are used to estimate the time span represented by a sequence of analogous rocks. When this time span is known from radiometric dates or fossils, however, it is almost always found that the modern analogue seriously underestimates it. Thus modern sedimentation rates are typically much higher than the accumulation rates of ancient rock sequences. Indeed, accumulation rates are known to decrease systematically as a function of time span in stratigraphical sections. The explanation appears to be that the strata of ancient rock reflect phases of erosion as well as deposition, and modern sedimentation rates may best be used as a guide to the time represented by a given layer of preserved rock. Thus the time span of specific layers expressed as a fraction of the total time span of the section gives a measure of the completeness of the record.

To the few empirical stratigraphical studies of time scale relationships, John Tipper (Nature 302, 696; 1983) has just added a very attractive statistical analysis, which integrates short-term sedimentological parameters and long-term stratigraphical phenomena. The attractiveness of Tipper's analysis is that it builds on a simple base that is consistent with present experience. He proposes to specify a sedimentation system in terms of the frequency distribution of its short-term rates of deposition. Negative rates represent erosion; and the mean, or expected, rate will be positive for a depositional regime and negative for an erosional regime. Longerterm accumulation can be modelled very simply from the frequency distribution by a random walk procedure, but it was not Tipper's objective merely to generate synthetic stratigraphical sections. He explores the general properties of longer-term accumulations.

The first result is that the expected longterm deposition rate is the same as the mean short-term rate, and not a function of time span. The empirical stratigraphical results appear to conflict with this finding, but this is because the two concepts of mean rate are not strictly analogous. Stratigraphical sections are the result of net accumulation and so represent only positive deposition rates. It follows that stratigraphical information cannot properly describe sedimentation systems.

Tipper investigates the probability that a time interval will be represented by any sediment accumulation. This probability is shown to be independent of the time span of the section, but to decrease significantly as the time interval becomes shorter. Thus, stratigraphical completeness should be ex pected to fall as greater precision is demanded.

Tipper's analysis also predicts the behaviour of stratigraphical completeness for a given level of precision. The level of precision is fixed by the time scale of the short-term sedimentation rates on which the model is based, and completeness is the fraction of the total time span of a section that is represented by the active deposition time of the preserved sediment increments. Thus defined, the expected value of completeness is shown to decrease systematically with increasing section duration.

The completeness of a stratigraphical section clearly limits the scope of interpretations that may justifiably be made from it, but is difficult to estimate because it is ultimately determined by a unique sedimentation regime. Can Tipper's analysis provide estimates of completeness from sedimentological information? Unfortunately, the time-scale factor indicates that it cannot, because if the short-term time units are short enough for modern observations, then the precision of the completeness estimate becomes far too fine for practical stratigraphy. Furthermore, the frequency distribution of sedimentation rates cannot be properly characterized unless the systems are observed for long enough to include rare events. This would mean integrating the evidence of stratigraphical sections, and Tipper has shown that the data are not compatible.

If we generalize the stratigraphical accumulation rates, however, the modern deposition rates can be made compatible by the simple expedient of ignoring negative values. Tipper's analysis now serves to indicate the properties of an acceptable estimator of completeness. The simple estimator of completeness, overall accumulation rate of a section divided by a shorter-term mean deposition rate for the same environment, meets the criterion that the expected value should fall when estimated at fiver precision. It succeeds because the empirical accumulation rates vary inversely with time span. The time scale of the short-term rates fixes the precision.

Such estimates of completeness can lead to counter-intuitive results. For example, our short-term experience of the episodic nature of fluvial deposition and the more uniform conditions of the shallow sea floor suggests that the river environment will produce less complete sections. At time scales longer than $10,000 \mathrm{yr}$, however, the similarity of stratigraphical accumulation rates dictates that we should not expect any difference on the basis of environment. Presumably, subsidence is more significant to the long-term accumulation than the short-term sedimentological processes that we can see.

Peter M. Sadler is in the Department of Earth Sciences, University of California, Riverside, California 92521. 